\title{
Targeted Sequencing Identifies the Genetic Variants Associated with High-altitude Polycythemia in the Tibetan Population
}

\author{
Zhiying Zhang ${ }^{1,2} \cdot$ Lifeng Ma ${ }^{1,2} \cdot$ Xiaowei Fan ${ }^{1,2} \cdot$ Kun Wang $^{3} \cdot$ Lijun Liu $^{1,2}$. \\ Yiduo Zhao ${ }^{1,2} \cdot$ ZhiPeng Zhao $^{1,2}$ - Han Zhang ${ }^{1,2}$ - Tian Liang ${ }^{1,2}$. Wenxue Dong ${ }^{1,2}$. \\ Peng Cai ${ }^{1,2}$ Yansong $\mathrm{Li}^{1,2} \cdot$ Jing $\mathrm{Li}^{1,2} \cdot$ Songhua $\mathrm{Zhou}^{4} \cdot$ Longli Kang $^{1,2}{ }_{(\mathbb{C})}$
}

Received: 5 April 2021/Accepted: 12 July 2021/Published online: 3 August 2021

(C) The Author(s) 2021

\begin{abstract}
High-altitude polycythemia (HAPC) is characterized by excessive proliferation of erythrocytes, resulting from the hypobaric hypoxia condition in high altitude. The genetic variants and molecular mechanisms of HAPC remain unclear in highlanders. We recruited 141 Tibetan dwellers, including 70 HAPC patients and 71 healthy controls, to detect the possible genetic variants associated with the disease; and performed targeted sequencing on 529 genes associated with the oxygen metabolism and erythrocyte regulation, utilized unconditional logistic regression analysis and GO (gene ontology) analysis to investigate the genetic variations of HAPC. We identified 12 single nucleotide variants, harbored in 12 genes, associated with the risk of HAPC $(4.7 \leq$ odd ratios $\leq 13.6$; $7.6 \mathrm{E}-08 \leq p$-value $\leq 1 \mathrm{E}-04)$. The pathway
\end{abstract}

Zhiying Zhang, Lifeng Ma, and Xiaowei Fan have contributed equally to this work.

Songhua Zhou

fszsh1001@163.com

$\triangle$ Longli Kang

longli_kang@163.com

1 Key Laboratory for Molecular Genetic Mechanisms and Intervention Research on High Altitude Disease of Tibet Autonomous Region, Xianyang, China

2 Key Laboratory of High Altitude Environment and Genes Related to Diseases of Tibet Autonomous Region, School of Medicine, Xizang Minzu University, Xianyang 712082, Shaanxi, China

3 Ministry of Education Key Laboratory of Contemporary Anthropology, Collaborative Innovation Center for Genetics and Development, School of Life Sciences, Fudan University, Shanghai 200433, China

4 Foshan University, Foshan 528000, Guangdong, China enrichment study of these genes indicated the three pathways, the PI3K-AKT pathway, JAK-STAT pathway, and HIF-1 pathway, are essential, which $p$-values as 3.70E $-08,1.28 \mathrm{E}-07$, and $3.98 \mathrm{E}-06$, respectively. We are hopeful that our results will provide a reference for the etiology research of HAPC. However, additional genetic risk factors and functional investigations are necessary to confirm our results further.

Keywords High-altitude polycythemia $\cdot$ Highland people $\cdot$ SNPs

\section{Introduction}

The Qinghai-Tibet Plateau, namely the "roof of the world," is a high-altitude area with an elevation between 3000 and $5000 \mathrm{~m}$. Hypoxia is one of the most critical characteristics of the high-altitude environment. Highaltitude polycythemia (HAPC) is one of the chronic highaltitude diseases developed in Tibetan dwellers, characterized by an excessive number of circulating erythrocytes. The clinical diagnosis of HAPC requires a hemoglobin concentration $(\mathrm{Hb})$ no less than $19 \mathrm{~g} / \mathrm{dL}$ for females and $21 \mathrm{~g} / \mathrm{dL}$ for males, respectively [1]. HAPC is often commonly accompanied by the symptoms, headache, dizziness, dyspnea, sleep disorders, or venous dilatation [1]. The incidence of HAPC in the Tibetan Plateau ranges from 5 to $18 \%$, increasing with altitude, a severe public health problem in China and other Andean countries [2].

The compensation for prolonged hypobaric hypoxia is the main reason for the change of hemoglobin concentration through elevating oxygen-carrying, transportation, and exchange [3]. Elevated hemoglobin concentrations are crucial for adapting to high altitude environment. However, 
the pathological increased red blood cell may trigger blood viscosity, potentially slowing blood flow and inciting hypercoagulation, thrombosis, or tissue hypoxia, even further aggravate various circulatory, respiratory, digestive, and neurological diseases [4-6]. While the etiology and the pathophysiological of HAPC remain unclear, the incidence of HAPC showed significantly individual differences.

Recent studies revealed that HAPC in Tibetans or Han is associated with several gene polymorphisms, such as phosphatidylinositol-4,5-bisphosphonate 3-kinase, catalytic subunit delta gene (PIK3CD), collagen type IV $\alpha 3$ chain gene (COL4A3), integrin subunit alpha six gene (ITGA6), erb-b2 receptor tyrosine kinase four gene (ERBB4), EPH receptor A2 gene (EPHA2), angiotensinogen gene (AGT), and endothelial PAS domain protein one gene (EPAS1) [7-11] EPHA2 can disturb erythropoiesis by regulating EPO production, and EPAS1 contributes to genetic adaptation to high altitudes and the particular trait of the low $\mathrm{Hb}$ concentrations, conditions frequently observed among Tibetans [9, 12]. HAPC was found to lead to morphological changes and pathological damage to the gastric mucosa of patients. In particular, the kallikrein gene cluster (KLK1, KLK3, KLK7, KLK8, and KLK12) was upregulated $>17$-fold. The elevated levels of KLK1, KLK3, KLK7, KLK8 and KLK12 may be closely associated with the hypertension, inflammation, obesity and other gastric injuries associated with polycythemia. Thus, the kallikreins are likely to potentially impact the development of HAPC [13]. Wang et al. [14] found that 19 serum proteins expressed differentially between patients and healthy controls with HAPC. Among them, C4A(The complement component 4A), C6(complement C6), CALR(Calreticulin), MASP1(Mannan Binding Lectin Serine Peptidase 1), and CNDP1(serum carnosinase) may enable researchers to use them as candidate plasma biomarkers for HAPC on account of their latent diagnostic, preventive and therapeutic values [14]. These results suggest that HAPC presents noticeable racial and significant individual differences in susceptibility.

In this study, we conducted a case-control study to investigate the association of genetic variants and the susceptibility of HAPC in the Tibetan population. We selected a group of genes considered involved in erythropoiesis and oxygen transportation by previous studies and using the target exome sequencing strategy to detect the coding variants. This work's overarching goal was to investigate associations between HAPC susceptibility and candidate genes that control oxygen metabolism in erythrocytes.

\section{Experimental Procedures}

\section{Study Populations}

HAPC was defined as males and females with $\mathrm{Hb} \geq 21 \mathrm{~g} /$ $\mathrm{dL}$ and $\mathrm{Hb} \geq 19 \mathrm{~g} / \mathrm{dL}$, respectively. We excluded individuals with chronic pulmonary diseases, lung cancer, or secondary polycythemia due to hypoxemia caused by certain chronic diseases. A total of 70 HAPC cases and 71 healthy controls of Tibetan inhabitants who lived in plateau areas above $2500 \mathrm{~m}$ were recruited from the Second People's Hospital of Tibet Autonomous Region. The cases include 35 males and 35 females. Control samples consisted of 39 males and 32 females. The age range of all samples was 20-60 years old. The Ethics Committee approved the study of the Xizang Minzu University with Ethics Number 201801.

\section{Epidemiological and Clinical Data}

Participants' information was collected through physicians or from medical chart review. All study participants provided written, informed consent for participation. Also, we obtained $5 \mathrm{ml}$ of peripheral blood specimens from each participant. All persons gave their informed consent before their inclusion in the study.

\section{Exome Sequencing and Bioinformatics of Target Genes}

Targeted exome sequencing (agilent All exon kit V5 $50 \mathrm{M}$ ) of 529 selected genes was conducted on all samples - these 529 genes with erythropoiesis and oxygen transportation by previous studies and database IPA (Ingenuity Pathway Analysis) and NCBI(National Center for Biotechnology Information), etc. [15]. Genomic DNA was extracted from the whole blood or saliva of individual subjects according to standard protocols. Double-stranded DNA was indexed and multiplexed in groups of six per lane and sequenced in 101-bp paired-end mode using the Illumina HiSeq 2000 platform. The mean on-target depth of each sample was 200X. The raw FASTQ files from the Illumina HiSeq were aligned to the human reference genome build 19 (GRCh37), and variants were called using SAMtools v0.1.18 and GATK v3.8. Low-quality variants were filtered as described previously [16]. Under the theory of "Common variants, common disease", we included the variants with minor allele frequency (MAF) $>0.05$ in the Asian population HapMap database into the subsequent analysis. 


\section{Statistical Analysis}

The genotype frequencies of each SNP in the control subjects were checked using the Hardy-Weinberg equilibrium (HWE). HWE values $>0.01$ were further analyzed. Genes FER (ferritin), CREB5(also called CREBPA, CRE (cyclic AMP response element)-binding proteins), and TP53 with HWE values $<0.01$ in the control group, failing to conform to the HWE rule, were excluded from the study.

The effects of polymorphisms on the risk of HAPC were expressed as odds ratios (ORs) with 95\% confidence intervals (95\% CIs), evaluated by three genetic models (dominant, recessive, and additive models) using unconditional logistic regression analysis. The Multiple comparisons were corrected by FDR and Bonferroni method. FDR and Bonferroni-corrected $p<0.05$ indicated a significant between-group difference. KEGG (Kyoto Encyclopedia of Genes and Genomes)-based enrichment analysis was conducted in R software [17]. All data were analyzed by R software package GenABEL20.

\section{Results}

Table 1 displays the demographics of patients with HAPC and controls. Table 2 and Fig. 1 show the significantly differentiated genes, using the unconditional logistic regression analysis, false discovery rate (FDR) calculation, and Bonferroni correction. We observed significant discrepancies between the HAPC case and control groups in the dominant and additive genetic models. The results indicated the 12 genes, PDK1, RUNDC3B, EPO, MET, PTK2, RELN, TDRD1, TCL1A, STAT3, STAT5A, IL12RB1, and $N F 2$, were associated with HAPC.

We adopted KEGG signaling pathways to determine gene-specific differences, and Table 3 and Fig. 2 display the results. Twelve genes were enriched in 16 signal pathways, including the PI3K-AKT pathway, JAK-STAT pathway, HIF-1 pathway, pathways in cancer, axon guidance, focal adhesion, proteoglycans in cancer, cytokinecytokine receptor interaction, et al. Enrichment pathway

Table 1 Demographics of healthy controls and patients with highaltitude polycythemia

\begin{tabular}{lll}
\hline Variables & \multicolumn{2}{l}{ Tibetan } \\
\cline { 2 - 3 } & Case $(\mathrm{n}=70)$ & Control $(\mathrm{n}=71)$ \\
\hline Male & 35 & 39 \\
Female & 35 & 32 \\
\hline
\end{tabular}

information is shown in Figs. 3 (according to Table 2, pvalue $<6.36 \mathrm{E}-05$ ) and 2 .

\section{Discussion}

\section{Genomics and Bioinformatics Enhance the Study of HAPC}

Upon completing the Human Genome Project, we will have a more comprehensive understanding of populationspecific genomic variations and the interactions between genes and environmental factors. This information will help us quickly determine mutation sites related to diseases to use genetic information to establish the relationship between sequence variation and disease risk and help prevent, diagnose, and treat diseases [18, 19].

HAPC seriously intimidates the fitness of the population of the plateau. In Tibet, the increased $\mathrm{Hb}$ concentration enhances the efficiency of carrying oxygen to adapt to altitude hypoxia. This reaction is an imperative factor for populations, who adapt to high altitudes. We found an increased risk of HAPC associated with SNPs in EPO, STAT3, PDK1, STAT5A, IL12RB1, PTK2, MET, TCL1A, RELN, RUNDC3B, TDRD1, and NF2 in the Tibetan population. We found that these genes were mostly enriched in the PI3K-AKT, JAK-STAT, and HIF-1 pathways through KEGG pathway analysis.

Notably, the genes' distributions varied widely from the different pathways based on the KEGG pathway analysis. From the perspective of the pathways, three genes $E P O$, STAT3, and PDK1 were enriched in HIF-1 pathways; four genes, EPO, STAT3, STAT5A, and ILI2RB1, were enriched in JAK-STAT pathways; five genes EPO, PTK2, MET, TCL1A, and RELN were enriched in PI3K-AKT pathways. Likewise, $E P O$ gene was enriched in these three pathways; STAT3 gene was enriched in two pathways; excluding $R U N D C 3 B, T D R D 1$, and $N F 2$ genes, other genes were positioned in one pathway.

\section{The Impact of Genes Enriched in the HIF-1 Pathway for Erythrocytes}

Hypoxia-inducible factor one (HIF-1), as a transcription factor, is composed of two subunits, which include an induced expressed part of HIF- $1 \alpha$ subunit and a control structure of HIF-1 $\beta$ subunit. Without hypoxia, HIF- $1 \alpha$ subunit would never be ubiquitinated and hydrolyzed by proteasomes easily [20]. Meanwhile, complex regulation of signal transduction cascades, which are mediated by cytokines and their homologous receptors, may affect the formation of HAPC. When the body is in a low-oxygen 
Table 2 Basic information on significant gene differences in highland people

\begin{tabular}{|c|c|c|c|c|c|c|c|c|c|c|c|c|c|c|}
\hline \multirow[t]{2}{*}{ SNP } & \multirow[t]{2}{*}{ Gene } & \multirow[t]{2}{*}{ CHR } & \multirow[t]{2}{*}{ Model } & \multirow{2}{*}{$\begin{array}{l}\text { Alleles } \\
\text { A/B }\end{array}$} & \multicolumn{3}{|c|}{ Case $(\mathrm{N})$} & \multirow{2}{*}{$\begin{array}{l}\text { Case } \\
\text { MAF }\end{array}$} & \multirow{2}{*}{\multicolumn{2}{|c|}{$\begin{array}{l}\text { HWE } \\
\text { Case }\end{array}$}} & \multicolumn{3}{|c|}{ Control (N) } & \multirow{2}{*}{$\begin{array}{l}\text { Control } \\
\text { MAF }\end{array}$} \\
\hline & & & & & AA & $\mathrm{AB}$ & $\mathrm{BB}$ & & & & AA & $\mathrm{AB}$ & $\mathrm{BB}$ & \\
\hline rs529091195 & PDK1 & 2 & Dominant & $/ \mathrm{T}$ & 0 & 48 & 20 & 0.3529 & $1.87 \mathrm{E}$ & -06 & 0 & 17 & 54 & 0.1197 \\
\hline rs529091195 & PDK1 & 2 & Additive & $/ \mathrm{T}$ & 0 & 48 & 20 & 0.3529 & $1.87 \mathrm{E}$ & -06 & 0 & 17 & 54 & 0.1197 \\
\hline rs 376837075 & FER & 5 & Dominant & $/ \mathrm{T}$ & 0 & 66 & 2 & 0.485 & $4.13 \mathrm{E}$ & -17 & 0 & 33 & 35 & 0.243 \\
\hline rs376837075 & FER & 5 & Additive & $/ \mathrm{T}$ & 0 & 66 & 2 & 0.485 & $4.13 \mathrm{E}$ & -17 & 0 & 33 & 35 & 0.243 \\
\hline rs527802276 & RUNDC3B & 7 & Dominant & /A & 0 & 61 & 7 & 0.449 & $8.42 \mathrm{E}$ & -13 & 0 & 29 & 41 & 0.2071 \\
\hline rs527802276 & RUNDC3B & 7 & Additive & /A & 0 & 61 & 7 & 0.449 & $8.42 \mathrm{E}$ & -13 & 0 & 29 & 41 & 0.2071 \\
\hline rs773485910 & EPO & 7 & Dominant & $\mathrm{T} /-$ & 0 & 46 & 22 & 0.338 & $6.99 \mathrm{E}$ & -06 & 0 & 22 & 49 & 0.155 \\
\hline rs773485910 & EPO & 7 & Additive & $\mathrm{T} /-$ & 0 & 46 & 22 & 0.338 & $6.99 \mathrm{E}$ & -06 & 0 & 22 & 49 & 0.155 \\
\hline rs397889442 & RELN & 7 & Dominant & $\mathrm{A} /-$ & 0 & 41 & 25 & 0.311 & $1.11 \mathrm{E}$ & -04 & 0 & 18 & 47 & 0.139 \\
\hline rs397889442 & RELN & 7 & Additive & $\mathrm{A} /-$ & 0 & 41 & 25 & 0.311 & $1.11 \mathrm{E}$ & -04 & 0 & 18 & 47 & 0.139 \\
\hline rs548208912 & CREB5 & 7 & Dominant & $\mathrm{T} /-$ & 0 & 61 & 3 & 0.477 & $8.11 \mathrm{E}$ & -15 & 0 & 33 & 34 & 0.246 \\
\hline rs548208912 & CREB5 & 7 & Additive & $\mathrm{T} /-$ & 0 & 61 & 3 & 0.477 & $8.11 \mathrm{E}$ & -15 & 0 & 33 & 34 & 0.246 \\
\hline rs551879100 & MET & 7 & Dominant & $\mathrm{T} /-$ & 0 & 58 & 10 & 0.427 & $6.20 \mathrm{E}$ & -11 & 0 & 33 & 38 & 0.232 \\
\hline rs551879100 & MET & 7 & Additive & $\mathrm{T} /-$ & 0 & 58 & 10 & 0.427 & $6.20 \mathrm{E}$ & -11 & 0 & 33 & 38 & 0.232 \\
\hline rs372806706 & PTK2 & 8 & Dominant & $\mathrm{A} /-$ & 0 & 61 & 6 & 0.455 & $3.86 \mathrm{E}$ & -13 & 0 & 31 & 39 & 0.221 \\
\hline rs372806706 & PTK2 & 8 & Additive & $\mathrm{A} /-$ & 0 & 61 & 6 & 0.455 & $3.86 \mathrm{E}$ & -13 & 0 & 31 & 39 & 0.221 \\
\hline rs369382658 & PTK2 & 8 & Dominant & $\mathrm{A} /-$ & 0 & 61 & 6 & 0.455 & $3.86 \mathrm{E}$ & -13 & 0 & 31 & 39 & 0.221 \\
\hline rs369382658 & PTK2 & 8 & Additive & $\mathrm{A} /-$ & 0 & 61 & 6 & 0.455 & $3.86 \mathrm{E}$ & -13 & 0 & 31 & 39 & 0.221 \\
\hline rs11285127 & TDRD1 & 10 & Dominant & $\mathrm{A} /-$ & 0 & 49 & 19 & 0.36 & $7.34 \mathrm{E}$ & -07 & 0 & 21 & 45 & 0.159 \\
\hline rs11285127 & TDRD1 & 10 & Additive & $\mathrm{A} /-$ & 0 & 49 & 19 & 0.36 & 7.34E & -07 & 0 & 21 & 45 & 0.159 \\
\hline rs142205645 & TCL1A & 14 & Dominant & /AG & 0 & 50 & 18 & 0.368 & $5.55 \mathrm{E}$ & -07 & 0 & 19 & 52 & 0.134 \\
\hline rs142205645 & TCL1A & 14 & Additive & /AG & 0 & 50 & 18 & 0.368 & $5.55 \mathrm{E}$ & -07 & 0 & 19 & 52 & 0.134 \\
\hline rs141204613 & TP53 & 17 & Dominant & /TTT & 1 & 64 & 3 & 0.485 & $1.49 \mathrm{E}$ & -14 & 0 & 35 & 31 & 0.265 \\
\hline rs141204613 & TP53 & 17 & Additive & /TTT & 1 & 64 & 3 & 0.485 & $1.49 \mathrm{E}$ & -14 & 0 & 35 & 31 & 0.265 \\
\hline rs558351915 & STAT3 & 17 & Dominant & $\mathrm{A} /-$ & 0 & 55 & 11 & 0.417 & $6.74 \mathrm{E}$ & -10 & 0 & 27 & 38 & 0.208 \\
\hline rs558351915 & STAT3 & 17 & Additive & $\mathrm{A} /-$ & 0 & 55 & 11 & 0.417 & $6.74 \mathrm{E}$ & -10 & 0 & 27 & 38 & 0.208 \\
\hline rs779456792 & STAT5A & 17 & Dominant & $\mathrm{T} /-$ & 0 & 52 & 16 & 0.382 & $5.58 \mathrm{E}$ & -08 & 0 & 26 & 45 & 0.183 \\
\hline rs779456792 & STAT5A & 17 & Additive & $\mathrm{T} /-$ & 0 & 52 & 16 & 0.382 & $5.58 \mathrm{E}$ & -08 & 0 & 26 & 45 & 0.183 \\
\hline rs548702753 & IL12RB1 & 19 & Dominant & $/ \mathrm{T}$ & 2 & 47 & 16 & 0.392 & $2.66 \mathrm{E}$ & -05 & 0 & 18 & 48 & 0.136 \\
\hline rs548702753 & IL12RB1 & 19 & Additive & $/ \mathrm{T}$ & 2 & 47 & 16 & 0.392 & $2.66 \mathrm{E}$ & -05 & 0 & 18 & 48 & 0.136 \\
\hline rs769771815 & NF2 & 22 & Dominant & $\mathrm{T} /-$ & 0 & 48 & 18 & 0.364 & $7.44 \mathrm{E}$ & -07 & 0 & 23 & 44 & 0.171 \\
\hline rs769771815 & NF2 & 22 & Additive & $\mathrm{T} /-$ & 0 & 48 & 18 & 0.364 & 7.44E & -07 & 0 & 23 & 44 & 0.171 \\
\hline \multirow[t]{2}{*}{ SNP } & \multirow[t]{2}{*}{ Gene } & \multirow[t]{2}{*}{ CHR } & \multirow[t]{2}{*}{ Model } & \multirow{2}{*}{\multicolumn{2}{|c|}{$\begin{array}{l}\text { HWE } \\
\text { Control }\end{array}$}} & \multicolumn{2}{|l|}{$95 \% \mathrm{CI}$} & \multirow{2}{*}{\multicolumn{2}{|c|}{$\mathrm{P}$}} & \multirow{2}{*}{\multicolumn{2}{|c|}{$\mathrm{P} 1$}} & $\mathrm{P} 2$ & & \\
\hline & & & & & & L95 & U95 & & & & & & & \\
\hline rs529091195 & PDK1 & 2 & Dominant & 0.583 & 7.624 & 3.585 & 16.21 & $1.31 \mathrm{E}$ & -07 & $8.74 \mathrm{E}$ & -05 & 0.000 & 026 & \\
\hline rs529091195 & PDK1 & 2 & Additive & 0.583 & 7.624 & 3.585 & 16.21 & $1.31 \mathrm{E}$ & -07 & $8.75 \mathrm{E}$ & -05 & 0.000 & 031 & \\
\hline rs376837075 & FER & 5 & Dominant & 0.007 & 35 & 7.928 & 15.45 & $2.70 \mathrm{E}$ & -06 & 0.000 & 179 & 0.008 & & \\
\hline rs376837075 & FER & 5 & Additive & 0.007 & 35 & 7.928 & 15.45 & $2.70 \mathrm{E}$ & -06 & 0.000 & & 0.008 & & \\
\hline rs527802276 & RUNDC3B & 7 & Dominant & 0.0312 & 12.32 & 4.933 & 30.77 & $7.58 \mathrm{E}$ & -08 & 0.000 & 874 & 0.000 & & \\
\hline rs527802276 & RUNDC3B & 7 & Additive & 0.0312 & 12.32 & 4.933 & 30.77 & $7.58 \mathrm{E}$ & -08 & 0.000 & 875 & 0.000 & 323 & \\
\hline rs773485910 & EPO & 7 & Dominant & 0.197 & 4.657 & 2.278 & 9.519 & $2.47 \mathrm{E}$ & -05 & 0.004 & & 0.075 & & \\
\hline rs773485910 & EPO & 7 & Additive & 0.197 & 4.657 & 2.278 & 9.519 & $2.47 \mathrm{E}$ & -05 & 0.004 & & 0.075 & & \\
\hline rs397889442 & RELN & 7 & Dominant & 0.593 & 4.282 & 2.05 & 8.944 & 0.000 & 087 & 0.019 & & 0.332 & & \\
\hline rs397889442 & RELN & 7 & Additive & 0.593 & 4.282 & 2.05 & 8.944 & 0.000 & 087 & 0.019 & & 0.333 & & \\
\hline rs548208912 & CREB5 & 7 & Dominant & 0.007 & 20.95 & 5.977 & 73.42 & $1.99 \mathrm{E}$ & -06 & 0.000 & 717 & 0.006 & & \\
\hline rs548208912 & CREB5 & 7 & Additive & 0.007 & 20.95 & 5.977 & 73.42 & $1.99 \mathrm{E}$ & -06 & 0.000 & 729 & 0.006 & & \\
\hline
\end{tabular}


Table 2 continued

\begin{tabular}{|c|c|c|c|c|c|c|c|c|c|c|}
\hline \multirow[t]{2}{*}{ SNP } & \multirow[t]{2}{*}{ Gene } & \multirow[t]{2}{*}{ CHR } & \multirow[t]{2}{*}{ Model } & \multirow{2}{*}{$\begin{array}{l}\text { HWE } \\
\text { Control }\end{array}$} & \multirow[t]{2}{*}{ OR } & \multicolumn{2}{|c|}{$95 \%$ CI } & \multirow[t]{2}{*}{$\mathrm{P}$} & \multirow[t]{2}{*}{ P1 } & \multirow[t]{2}{*}{$\mathrm{P} 2$} \\
\hline & & & & & & L95 & U95 & & & \\
\hline rs551879100 & MET & 7 & Dominant & 0.015 & 6.679 & 2.95 & 15.12 & $5.26 \mathrm{E}-06$ & 0.001239 & 0.01611 \\
\hline rs551879100 & MET & 7 & Additive & 0.015 & 6.679 & 2.95 & 15.12 & $5.26 \mathrm{E}-06$ & 0.001241 & 0.01613 \\
\hline rs372806706 & PTK2 & 8 & Dominant & 0.016 & 12.79 & 4.887 & 33.47 & $2.08 \mathrm{E}-07$ & 0.0001061 & 0.0006368 \\
\hline rs372806706 & PTK2 & 8 & Additive & 0.016 & 12.79 & 4.887 & 33.47 & $2.08 \mathrm{E}-07$ & 0.0001063 & 0.0006377 \\
\hline rs369382658 & PTK2 & 8 & Dominant & 0.016 & 13.62 & 4.463 & 41.58 & $4.49 \mathrm{E}-06$ & 0.001205 & 0.01376 \\
\hline rs369382658 & PTK2 & 8 & Additive & 0.016 & 13.62 & 4.463 & 41.58 & $4.49 \mathrm{E}-06$ & 0.001206 & 0.01378 \\
\hline rs11285127 & TDRD1 & 10 & Dominant & 0.344 & 5.526 & 2.634 & 11.59 & $6.11 \mathrm{E}-06$ & 0.001337 & 0.01871 \\
\hline rs11285127 & TDRD1 & 10 & Additive & 0.344 & 5.526 & 2.634 & 11.59 & $6.11 \mathrm{E}-06$ & 0.001338 & 0.01874 \\
\hline rs142205645 & TCL1A & 14 & Dominant & 0.345 & 7.602 & 3.582 & 16.13 & $1.27 \mathrm{E}-07$ & $8.74 \mathrm{E}-05$ & 0.000389 \\
\hline rs142205645 & TCL1A & 14 & Additive & 0.345 & 7.602 & 3.582 & 16.13 & $1.27 \mathrm{E}-07$ & $8.75 E-05$ & 0.0003895 \\
\hline rs141204613 & TP53 & 17 & Dominant & 0.003 & 19.19 & 5.475 & 67.27 & $3.90 \mathrm{E}-06$ & 0.001195 & 0.01195 \\
\hline rs141204613 & TP53 & 17 & Additive & 0.003 & 19.14 & 5.477 & 66.85 & $3.75 \mathrm{E}-06$ & 0.001151 & 0.01151 \\
\hline rs558351915 & STAT3 & 17 & Dominant & 0.054 & 7.037 & 3.118 & 15.88 & $2.62 \mathrm{E}-06$ & 0.0009179 & 0.008021 \\
\hline rs558351915 & STAT3 & 17 & Additive & 0.054 & 7.037 & 3.118 & 15.88 & $2.62 \mathrm{E}-06$ & 0.0009191 & 0.008031 \\
\hline rs779456792 & STAT5A & 17 & Dominant & 0.105 & 5.625 & 2.685 & 11.79 & $4.72 \mathrm{E}-06$ & 0.001205 & 0.01446 \\
\hline rs779456792 & STAT5A & 17 & Additive & 0.105 & 5.625 & 2.685 & 11.79 & $4.72 \mathrm{E}-06$ & 0.001206 & 0.01447 \\
\hline rs548702753 & IL12RB1 & 19 & Dominant & 0.592 & 8.003 & 3.688 & 17.37 & $1.43 \mathrm{E}-07$ & $8.74 \mathrm{E}-05$ & 0.0004371 \\
\hline rs548702753 & IL12RB1 & 19 & Additive & 0.592 & 8.003 & 3.688 & 17.37 & $1.43 \mathrm{E}-07$ & $8.75 \mathrm{E}-05$ & 0.0004375 \\
\hline rs769771815 & NF2 & 22 & Dominant & 0.189 & 5.101 & 2.434 & 10.69 & $1.59 \mathrm{E}-05$ & 0.003255 & 0.04882 \\
\hline rs769771815 & NF2 & 22 & Additive & 0.189 & 5.101 & 2.434 & 10.69 & $1.59 \mathrm{E}-05$ & 0.003259 & 0.04889 \\
\hline
\end{tabular}

SNP single-nucleotide polymorphism, MAF minor allele frequency, HWE Hardy-Weinberg equilibrium, OR odds ratio, 95\% CI 95\% confidence interval, P P value calculated by unconditional logistic regression analysis, P1 P value FDR-calculated, P2 P value after Bonferroni correction

Fig. 1 Manhattan plot of the p-value of the correlation between HAPC and SNP determined by false discovery rate (FDR) calculation
Manhattan Plot

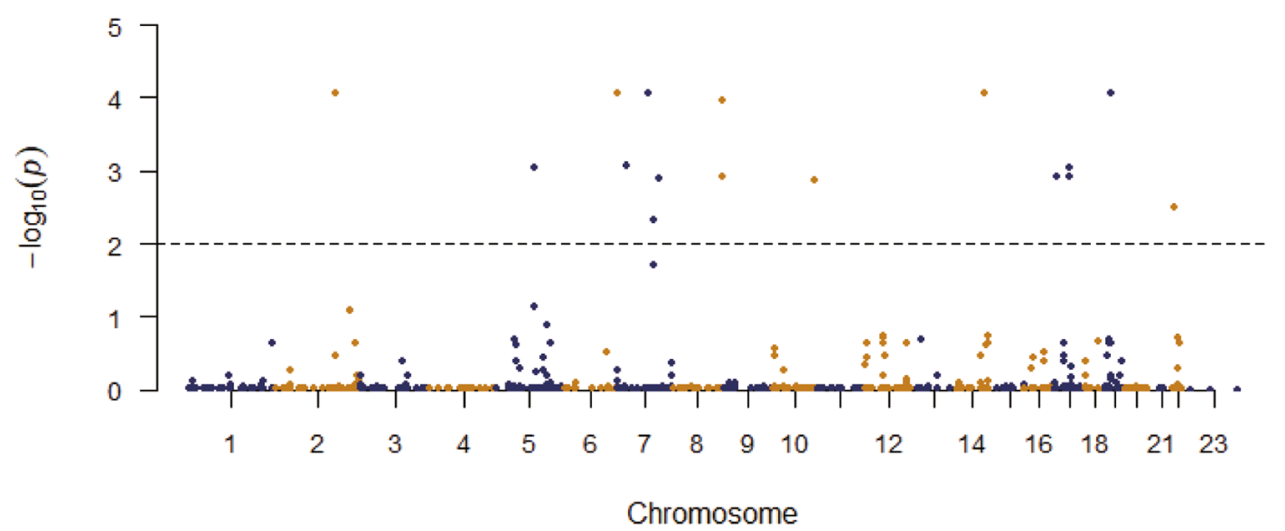

environment, its red blood cells increase. The HIF-1 signaling pathway may activate this.

$E P O$ is the earliest hypoxic adaptation gene, and its polymorphism is related to the formation of HAPC [21]. Our results conformed these findings as we found that the rs773485910 of the EPO gene was associated with an increased prevalence of HAPC. EPO, endogenous glycoprotein hormone, has a molecular weight of $39 \mathrm{KD}$, with 166 residues. It is a member of the hematopoietic cytokine family and is primarily involved in erythropoiesis. EPO is principally secreted by liver cells in infancy and by kidney cells in adulthood. When the body immerses in a low- 
Table 3 KEGG pathway analysis of differentially expressed genes

\begin{tabular}{|c|c|c|c|c|c|}
\hline Pathway & $\begin{array}{l}\text { KEGG } \\
\text { ID }\end{array}$ & $\begin{array}{l}\text { Input } \\
\text { number }\end{array}$ & $\begin{array}{l}\text { Background } \\
\text { number }\end{array}$ & $p$-value & Genes \\
\hline PI3K-Akt signaling pathway & hsa04151 & 5 & 342 & $3.70 \mathrm{E}-08$ & $\begin{array}{l}\text { PTK2, MET, TCL1A } \\
\text { RELN, EPO }\end{array}$ \\
\hline Jak-STAT signaling pathway & hsa04630 & 4 & 158 & $1.28 \mathrm{E}-07$ & $\begin{array}{l}\text { IL12RB1, STAT5A, } \\
\text { STAT3, EPO }\end{array}$ \\
\hline HIF-1 signaling pathway & hsa04066 & 3 & 103 & $3.98 \mathrm{E}-06$ & PDK1, STAT3, EPO \\
\hline Pathways in cancer & hsa05200 & 4 & 397 & $4.73 \mathrm{E}-06$ & $\begin{array}{l}\text { PTK2, STAT5A, } \\
\text { MET, STAT3 }\end{array}$ \\
\hline Axon guidance & hsa04360 & 3 & 176 & $1.91 \mathrm{E}-05$ & PDK1, MET, PTK2 \\
\hline Focal adhesion & hsa04510 & 3 & 203 & $2.91 \mathrm{E}-05$ & RELN, MET, PTK2 \\
\hline Proteoglycans in cancer & hsa05205 & 3 & 205 & $3.00 \mathrm{E}-05$ & PTK2, MET, STAT3 \\
\hline Cytokine-cytokine receptor interaction & hsa04060 & 3 & 265 & $6.36 \mathrm{E}-05$ & IL12RB1, MET, EPO \\
\hline Inflammatory bowel disease (IBD) & hsa05321 & 2 & 66 & $1.880 \mathrm{E}-04$ & IL12RB1, STAT3 \\
\hline Central carbon metabolism in cancer & hsa05230 & 2 & 67 & $1.936 \mathrm{E}-04$ & STAT5A, IL12RB1 \\
\hline Prolactin signaling pathway & hsa04917 & 2 & 72 & $2.227 \mathrm{E}-04$ & STAT5A, STAT3 \\
\hline Bacterial invasion of epithelial cells & hsa05100 & 2 & 78 & $2.603 \mathrm{E}-04$ & MET, PTK2 \\
\hline ErbB signaling pathway & hsa04012 & 2 & 88 & $3.293 \mathrm{E}-04$ & STAT5A, PTK2 \\
\hline AGE-RAGE signaling pathway in diabetic complications & hsa04933 & 2 & 101 & $4.310 \mathrm{E}-04$ & STAT5A, STAT3 \\
\hline
\end{tabular}

The above results were analyzed using DAVID and KOBAS online analysis tools

PI3K-Akt: Phosphatidylinositol 3 kinase(PI3K) /protein kinase B(AKT), Jak-STAT: Janus kinase (JAK)/ signal transducer and activator of transcription (STAT), HIF-1: Hypoxia inducible factor-1 (HAF-1), ErbB: Receptor tyrosine-protein kinase erbB-2, i.e. Her2, human epidermal growth factor receptor 2 (HER2), AGE-RAGE: advanced glycation end products (AGE) / receptor for dvanced glycation end products (RAGE)

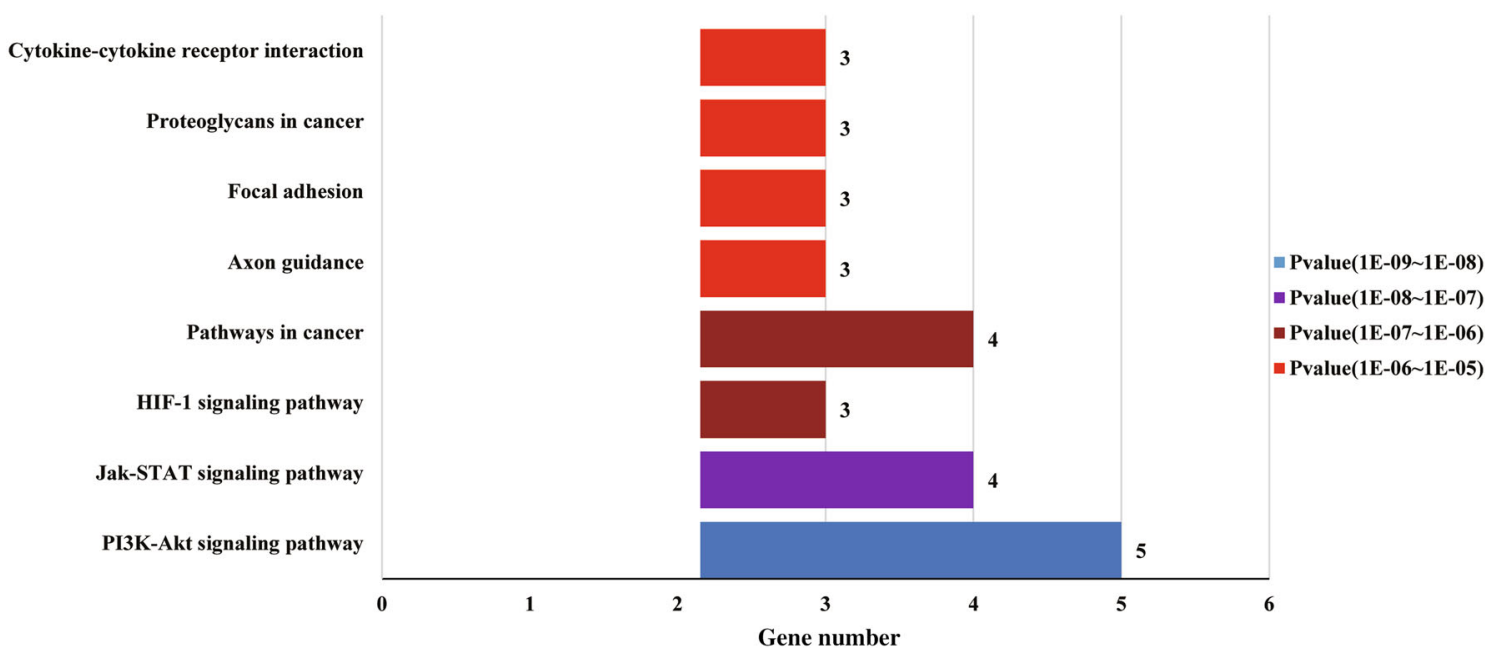

Fig. 2 KEGG pathway analysis of differentially expressed genes. The Y-axis is the name of the KEGG metabolic pathway, and the X-axis is the number of genes annotated to the pathway (according to Table 2, $p$-value $<6.36 \mathrm{E}-05$ )

oxygen environment, the acetylation level of HIF-1 alpha increase, and there is enhanced transcriptional activation of a series of target genes, stimulating increased secretion of EPO by the kidneys [22]. Via binding to the EPO receptor (EPOR) on red cell progenitors in the bone marrow, circulating EPO can arouse an increase in the amount of erythrocytes [21]. However, small amounts are also expressed in other vital organs, such as the brain, spleen, lungs, testicles, and placental tissue. At average oxygen concentrations, the EPO content of blood is low, and its primary role relates to the renewal of aging erythrocytes [23].

PDKs are serine protein kinase genes located on chromosome 2, mainly expressed in the heart, bone marrow, 
Fig. 3 Schematic diagrams of the JAK-STAT pathway, HIF-1 pathway, and PI3K-AKT pathway. The HIF-1 signaling pathway is activated, and EPO secretion increases under hypoxic conditions. EPO then binds to the EPO receptor (EPOR). The JAK-STAT signaling pathway can also be activated and plays an antiapoptotic role. The PI3K-Akt signaling pathway could promote the expression of the anti-apoptotic gene Bcl-xL and affect cell proliferation and differentiation. TCL1A activates the AKT pathway, which plays a role in cell survival by affecting the transcriptional activity of nuclear factor-kB (NFkB)

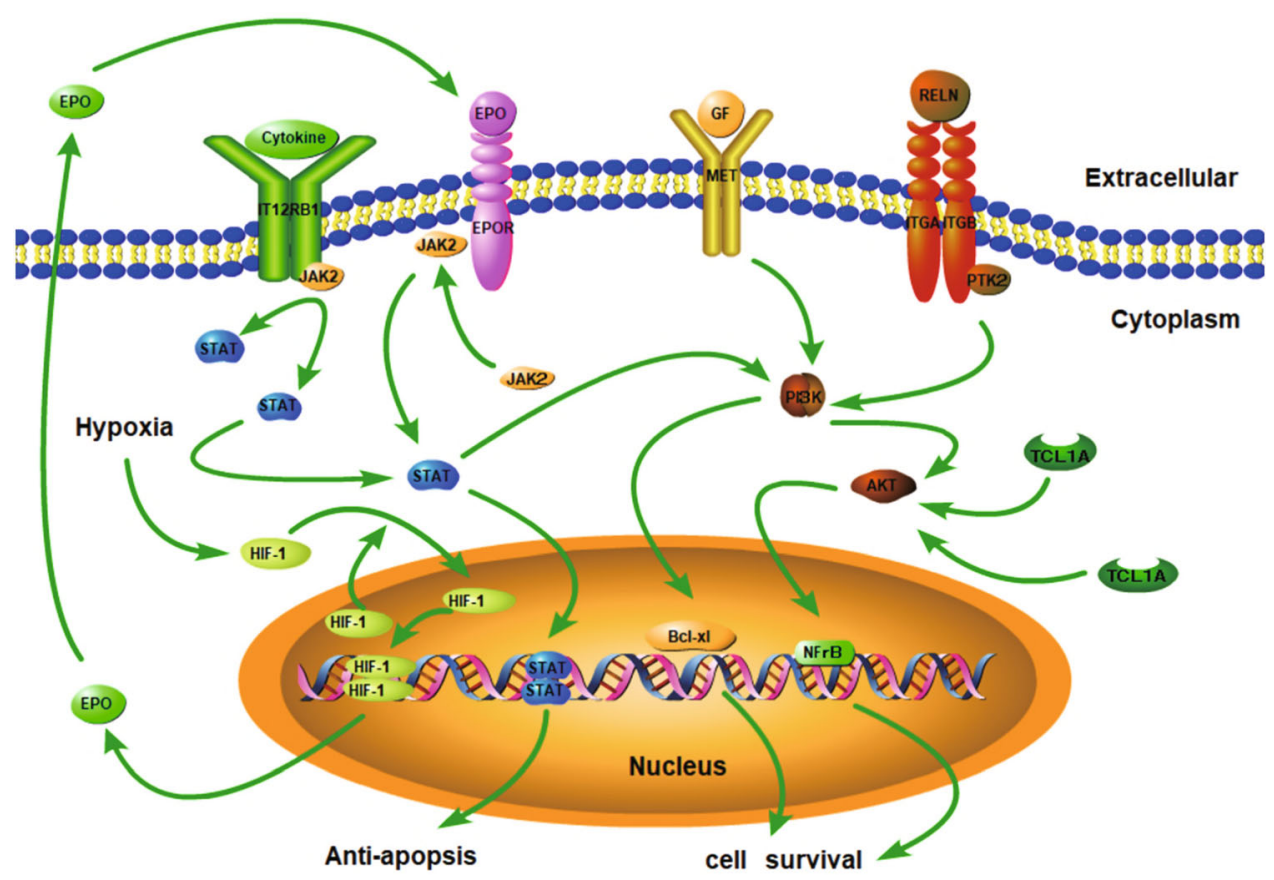

kidneys, and skin. There are four isomers of PDK-PDK1, PDK2, PDK3, and PDK4. PDK1, PDK2, and PDK3 are mainly involved in cellular glucose metabolism, while PDK4 is engaged primarily in cell fat metabolism. Previous studies have shown that PDK1 and PDK4 genes are associated with erythrocyte polymorphism in Chuvash decent people. Our research found that rs529091195 of the PDK gene was associated with HAPC, which might be caused by significant upregulation of HIF due to chronic hypoxia. Upregulated HIF can promote PDK1 expression, affecting the anaerobic oxidation of cells and providing energy for normal cellular life activities [24].

Moreover, by retrieving PDK1 in the KEGG pathway database, we acquire that PDK1 also brings a crucial impact in the PI3K-AKT pathway and is activated by plasma membrane intrinsic protein 3 (PIP3). Activated PDK1 fully activates adjacent protein kinase B (AKT) and regulates downstream AGC family protein kinase activity [25-27]. These actions allow PDK1 to control the physiological effects of insulin and growth factors, increase glucose uptake, promote glycogen and protein synthesis, and provide energy for cell proliferation and differentiation [28]. In this way, PDK1 influences the development of HAPC. Once activated, the PI3K-AKT pathway can also play an anti-apoptotic role, resulting in increased erythrocyte accumulation and promoting HAPC [11].

\section{The Impact of Genes Enriched in the JAK-STAT Pathway for Erythrocytes}

Important hematological factors widely involve the JAKSTAT pathway. When EPO binds to the EPO receptor, the JAK-STAT signaling pathway can also be activated [29]. Among those principal kinases involved in mediating EPOresponsive signal transduction, the Jak2 protein tyrosine kinase was identified for the first time by the researchers. JAK2 binds to EPOR at the bottom of the cytoplasm, causing JAK2 phosphorylation, which leads to tyrosine phosphorylation and coupling of STAT-5, affecting cell proliferation and differentiation [30].

STATs consist of seven separate members, STAT1, STAT2, STAT3, STAT4, STAT5A, STAT5B, and STAT6. Furthermore, they are active in many cell signaling pathways and have essential effects on innate immunity, acquired immunity, cell proliferation, differentiation, and survival [31]. We found that rs558351915 of the STAT3 gene and rs779456792 of the STAT5A gene were related to the formation of HAPC. Previous studies have shown that different STAT family members can interact in cell signaling pathways to regulate target genes' expression [32]. The JAK-STAT pathway plays an imperative role in cellular erythropoiesis, proliferation, and differentiation. STAT3 and STAT5 are essential phosphorylated kinases in the JAK-STAT cell signaling pathway. Meanwhile, EPO binds to the EPO receptor to activate and phosphorylated JAK2; and tyrosine phosphorylated and coupled with STAT5. Then, JAK1 and STAT5 upregulate the expression of membrane proteins, cytoskeleton, hematopoietic growth 
factor-related genes, and downstream target genes, which contain anti-apoptotic genes $\mathrm{Bcl}-\mathrm{xl}$ and $\mathrm{Bcl}-2$. Moreover, the PI3K-AKT pathway is a downstream effector of JAK2STAT5 signaling. Also, STAT5 regulates and promotes hemoglobin expression, thereby induces the proliferation of red blood cells (RBC). In a word, it can increase the RBCs' count in the body's blood [33].

\section{The Impact of Genes Enriched in the PI3K-AKT Pathway for Erythrocytes}

The PI3K-Akt signaling pathway is also called the "cell survival signaling pathway," which can protect cells from inactivation and affect cell proliferation and differentiation by being activated. This signaling pathway brings a substantial impact on the process of erythropoiesis and could downregulate apoptosis by regulating apoptosis-related molecules. This process promotes the expression of the anti-apoptotic gene $B c l-x L$ and plays a critical anti-apoptotic role [34]. In particular, PI3K-AKT pathways affect HIF-1 $\alpha$ transcriptional activity in hypoxia $[35,36]$, sequentially transactivating EPO, eventually resulting in erythrocytosis. Therefore, the PI3K-AKT signal pathway appeared to be involved in the mechanism of decreased erythroblasts apoptosis.

PTK2 genes are enriched in the PI3K-AKT signaling pathways. Upon cell-extracellular matrix (cell-ECM) contact, PTK2 can be recruited into focal plaques and is rapidly autophosphorylated to recruit other scaffolds and signal molecules to activate the downstream PI3K-AKT signaling pathways [37]. Studies also indicated that HIF-1 gene knockout could effectively inhibit the accumulation of HIF-1 protein and the expression of PTK2 mRNA. Meanwhile, this study also indicated that PTK2 activation was inhibited; the phosphorylation levels of downstream AKT were significantly reduced. These reductions indicated that PTK2 induced the phosphorylation of AKT. The transcriptional activation of PTK2, mediated by HIF-1, protects cells from inactivation and can affect cells' proliferation and differentiation by activating the cell survival signaling pathways and inhibiting the pro-apoptotic signaling pathways.

MET gene is enriched in the PI3K-AKT signaling pathways, located on chromosome 7. Its product is a sort of receptor tyrosine kinase of proteins, with about $110 \mathrm{kB}$ in size. MET is mainly expressed in the liver and kidneys, bone marrow cells. The mature MET protein is transmembrane, a dimer complex composed of $\alpha$ and $\beta$ subunits. There are three structural regions, of which the intracellular domain contains the binding sites for many signal molecules MET activation of the PI3K-Akt signaling pathways can promote cell proliferation and prevent cell apoptosis $[38,39]$. In addition to activating the above signaling pathways, MET can also interact with cell death receptors on cell membranes to play an anti-apoptotic role (eg. Fas, FasL). These findings illustrate that MET receptors play a direct role in preventing apoptosis.

\section{RUNDC3B, TDRD1 and NF2 Genes Influence Erythrocytes}

RUNDC3B (RUN domain containing 3B) is located on chromosome 7 and is widely expressed in the adrenal glands, brain, liver, small intestines, and other tissues. Although the biological function of RUNDC3B has not been determined, decreased expression of RUNDC3B may result in lymphoid malignancies [40]. In our study, we indicate that the rs527802276 of the RUNDC3B gene was associated with HAPC. Studies have shown that RUNDC3B and RUNDC3A (a Rap2-interacting protein) have high homology, and RUNDC3B also has a similar integration effect like RUNDC3A. The Rap protein family constitutes a subgroup of the Ras superfamily and works as a molecular "switch" regulating various cell functions, such as proliferation, differentiation, and other cell activities [41]. RUNDC3B contains a RUN domain in its $\mathrm{N}$-terminal region, which is an essential component of the mitogen-activated protein kinase (MAPK) cascade. Furthermore, Rap2 interacts with MAP4K4 through its C-terminal citron homology domain. MAP4K4 is a kind of the STE20 protein kinases and regulates c-Jun N-terminal kinase (JNK). Rap2 enhances MAP4K4-induced activation of JNK. So, RUNDC3B appears to play an essential role in activating c-JUN and c-Fos transcription factors, leading to the expression of c-JUN and c-Fos in the nucleus. Its product AP1 can bind to DNA sequences and induce cell proliferation and differentiation.

The TDRD1 gene on chromosome 10 is mainly expressed in the prostate and testes, and a small amount is expressed in the kidneys. Studies have shown that TDRD1 is involved in spermatogenesis. The mutation of the TDRD1 gene is related to spermatogenesis disorder in Han males [42]. In our study, rs11285127 of the TDRD1 gene was involved in the development of HAPC.

NF2 is located on chromosome 22, and its coding product is Merlin. It has a protein with a similar structure to ERM family members. Mutation of the gene is associated with neurofibromatosis type II [43]. In our study, the rs779456792 locus of the NF2 gene was associated with HAPC. The Merlin protein may regulate the PI3K-Akt signaling pathway by interacting with PI3K and other molecules. In this way, the Merlin protein controls cell survival, proliferation, differentiation, and development of $\mathrm{RBC}$, thereby affecting the formation of RBCs [44]. 


\section{Conclusion}

PDK1 (rs529091195), RUNDC3B (rs527802276), EPO (rs773485910), RELN (rs397889442), MET (rs551879100), TDRD1 (rs11285127), PTK2 (rs372806706, rs369382658), TCL1A (rs142205645), STAT3 (rs558351915), STAT5A (rs779456792), IL12RB1 (rs548702753) and NF2 (rs779456792) genes are associated with increased risk of HAPC. Through KEGG pathway analysis, we found that these genes were mostly enriched in the PI3K-AKT, JAKSTAT, and HIF-1 pathways.

We are hopeful that our results will provide a reference for the etiology research of HAPC. However, additional genetic risk factors and functional investigations are necessary to confirm our results further.

Author Contribution L.K. and S.Z. conceived, designed the study, supervised the project, and drafted the manuscript. Z.Z., L.M., X.F., K.W., participated in the design of the study, data analysis and helped to draft the manuscript. Z.Z., L.L., Y.Z., P.C., Y.L., H.Z., T.L., W.D., Z.Z., J.L., contributed to sample collection and experiments. And the manuscript has been reviewed and approved by all co-authors before submission.

Funding This study was supported by the National Natural Science Foundation of China (No. U20A20395, 81860329, 31660307, 31460286,31260252 ), the Natural Science Foundation of Xizang (Tibet) Autonomous Region (Nos. XZ201801-GB03, Z2014A09G23 ), the Science and Technology Department Project of Tibet Autonomous Region [Nos. XZ 2019 ZRG-32(Z); XZ 2019 ZRG-132; XZ2019ZRG-133; XZ2019ZRG-28(Z)], the Peak Scholars Programme of Xizang Minzu University, the Innovation Support Program for Young Teachers of Tibet Autonomous Region (Nos. QCZ2016-27; QCZ2016-29; QCZ2016-34).

\section{Declarations}

Conflict of interest The authors declare no competing financial interests.

Ethics Approval The study was approved by the Ethics Committee of the Xizang Minzu University. Number: 201801.

Consent to Participate All the volunteers signed an informed consent.

Consent for Publication All the authors approved to publish.

Supplementary Information The online version contains supplementary material available at https://doi.org/10.1007/s12288021-01474-1.

Open Access This article is licensed under a Creative Commons Attribution 4.0 International License, which permits use, sharing, adaptation, distribution and reproduction in any medium or format, as long as you give appropriate credit to the original author(s) and the source, provide a link to the Creative Commons licence, and indicate if changes were made. The images or other third party material in this article are included in the article's Creative Commons licence, unless indicated otherwise in a credit line to the material. If material is not included in the article's Creative Commons licence and your intended use is not permitted by statutory regulation or exceeds the permitted use, you will need to obtain permission directly from the copyright holder. To view a copy of this licence, visit http://creativecommons. org/licenses/by/4.0/.

\section{References}

1. Leon VF, Maggiorini M, Reeves JT et al (2005) Consensus statement on chronic and subacute high altitude diseases. High Alt Med Biol 6:147-157

2. Luks AM, Swenson ER, Bartsch P (2017) Acute high-altitude sickness. Eur Respir Rev 143:160096-160099

3. Palmer BF (2010) Physiology and pathophysiology with ascent to altitude. Am J Med Sci 1:69-77

4. Guan W, Ga Q, Li R et al (2015) Sleep disturbances in long-term immigrants with chronic mountain sickness: a comparison with healthy immigrants at high altitude. Respir Physiol Neurobiol 206:4-10

5. Jiang C, Liu F, Luo Y et al (2012) Gene expression profiling of high altitude polycythemia in Han Chinese migrating to the Qinghai-Tibetan plateau. Mol Med Rep 1:287-293

6. Reeves JT, Leon-velarde F (2004) Chronic mountain sickness: recent studies of the relationship between hemoglobin concentration and oxygen transport. High Alt Med Biol 2:147-155

7. Chen Y, Jiang C, Luo Y et al (2014) An EPAS1 haplotype is associated with high altitude polycythemia in male Han Chinese at the Qinghai-Tibetan plateau. Wilderness Environ Med 4:392-400

8. Fan X, Ma L, Zhang Z et al (2018) Associations of high-altitude polycythemia with polymorphisms in PIK3CD and COL4A3 in Tibetan populations. Hum Genomics 1:37-47

9. Liu L, Zhang Y, Zhang Z et al (2017) Associations of high altitude polycythemia with polymorphisms in and in Chinese Han and Tibetan populations. Oncotarget 8:53234-53243

10. Xu J, Yang YZ, Tang F et al (2015) EPAS1 gene polymorphisms are associated with high altitude polycythemia in Tibetans at the Qinghai-Tibetan plateau. Wilderness Environ Med 3:288-294

11. Zhao C, Li Z, Ji L et al (2017) PI3K-Akt signal transduction tolecules maybe involved in downregulation of erythroblasts apoptosis and perifosine increased its apoptosis in chronic mountain sickness. Med Sci Monit 23:5637-5649

12. Beall CM, CavalleriA GL, Deng L et al (2010) Natural selection on EPAS1 (HIF2alpha) associated with low hemoglobin concentration in Tibetan highlanders. Proc Natl Acad Sci USA 25:11459-11464

13. Li K, Gesang L, Dan Z et al (2017) Transcriptome reveals the over expression of a kallikrein gene cluster (KLK1/3/7/8/12) in the Tibetans with high altitude-associated polycythemia. Int $\mathbf{J}$ Mol Med 2:287-296

14. Wang Z, Liu F, Ye S et al (2019) Plasma proteome profiling of high-altitude polycythemia using TMT-based quantitative proteomics approach. J Proteomics 194:60-69

15. Tumuru L, Thein SL (2017) Genetic control of erythropoiesis. Curr Opin Hematol 3:173-182

16. Warden C D, Adamson A W, Neuhausen, S L, \& Wu X. Detailed comparison of two popular variant calling packages for exome and targeted exon studies. PeerJ, 2014; 2, e600.

17. Yu G, Wang LG, Han Y et al (2012) clusterProfiler: an R package for comparing biological themes among gene clusters. OMICS 5:284-287

18. Collins FS, Morgan M, Patrinos A (2003) The Human Genome Project: lessons from large-scale biology. Science 300:286-290 
19. Watson JD (1990) The human genome project: past, present, and future. Science 248:44-49

20. Weidemann A, Johnson RS (2008) Biology of HIF-1alpha. Cell Death Differ 4:621-627

21. Lappin T R, Lee F S. Update on mutations in the HIF: EPO pathway and their role in erythrocytosis. Blood Rev, 2019; 37: 100590

22. Tanaka T, Nangaku M (2012) Recent advances and clinical application of erythropoietin and erythropoiesis-stimulating agents. Exp Cell Res 9:1068-1073

23. Buroker NE, Ning XH, Zhou ZN et al (2010) Genetic associations with mountain sickness in Han and Tibetan residents at the Qinghai-Tibetan Plateau. Clin Chim Acta 19:1466-1473

24. Formenti F, Constantin TD, Emmanuel Y et al (2010) Regulation of human metabolism by hypoxia-inducible factor. Proc Natl Acad Sci USA 28:12722-12727

25. Lu Z, Coxhipkin MA, Windsor WT et al (2010) 3-phosphoinositide-dependent protein kinase-1 regulates proliferation and survival of cancer cells with an activated mitogen-activated protein kinase pathway. Mol Cancer Res 3:421-432

26. Mora A, Komander D, Van Aalten MF et al (2004) PDK1, the master regulator of AGC kinase signal transduction. Semin Cell Dev Biol 2:161-170

27. Parrales A, Lopez E, Colome AM (2011) Thrombin activation of PI3K/PDK1/Akt signaling promotes cyclin D1 upregulation and RPE cell proliferation. Biochim Biophys Acta 10:1758-1766

28. Knight ZA (2011) For a PDK1 inhibitor, the substrate matters. Biochem J 2:e1-e2

29. Ma S, Chen J, Chen C et al (2018) Erythropoietin rescues memory impairment in a rat model of chronic cerebral hypoperfusion via the EPO-R/JAK2/STAT5/PI3K/Akt/GSK-3 $\beta$ pathway. Mol Neurobiol 4:3290-3299

30. Rane SG, Reddy EP (2002) JAKs, STATs and Src kinases in hematopoiesis. Oncogene 21:3334-3358

31. Ivashkiv LB, Hu X (2004) Signaling by STATs. Arthritis Res Ther 4:159-168

32. Granillo A R, Boffi J C, BaranaoL, et al (2007) STAT5 transcriptional activity is impaired by LIF in a mammary epithelial cell line. Biochem Biophys Res Commun 3: 727-732

33. Buitenhuis M, Coffer PJ, Koenderman L (2004) Signal transducer and activator of transcription 5 (STAT5). Int J Biochem Cell Biol $11: 2120-2124$
34. Sun G, Zhou Y, Li H et al (2013) Over-expression of microRNA494 up-regulates hypoxia-inducible factor-1 alpha expression via PI3K/Akt pathway and protects against hypoxia-induced apoptosis. J Biomed Sci 20:100

35. Kirito K (2011) Regulation of erythropoiesis by hypoxia inducible factors (HIFs). Rinsho Ketsueki 6:368-375

36. Zhou J, Schmid T, Frank R et al (2004) PI3K/Akt is required for heat shock proteins to protect hypoxia-inducible factor 1alpha from pVHL-independent degradation. $\mathrm{J}$ Biol Chem 14:13506-13513

37. Bouchard V, Demers MJ, Thibodeau S et al (2007) Fak/Src signaling in human intestinal epithelial cell survival and anoikis: differentiation state-specific uncoupling with the PI3-K/Akt-1 and MEK/Erk pathways. J Cell Physiol 3:717-728

38. Chappell WH, Steelman LS, Long JM et al (2011) Ras/Raf/MEK/ ERK and PI3K/PTEN/Akt/mTOR inhibitors: rationale and importance to inhibiting these pathways in human health. Oncotarget 3:135-164

39. Zhang Y, Xia M, Jin K et al (2018) Function of the c-Met receptor tyrosine kinase in carcinogenesis and associated therapeutic opportunities. Mol Cancer 1:45-49

40. Burmeister DW, Smith EH, Cristel RT et al (2017) The expression of RUNDC3B is associated with promoter methylation in lymphoid malignancies. Hematol Oncol 1:25-33

41. Rountree MR, Bachman KE, Herman JG et al (2001) DNA methylation, chromatin inheritance, and cancer. Oncogene 24:3156-3165

42. Zhu XB, Lu JQ, Zhi EL et al (2016) Association of a TDRD1 variant with spermatogenic failure susceptibility in the Han Chinese. J Assist Reprod Genet 8:1099-1104

43. Giovannini M, Robanus ME, Vander VM et al (2000) Conditional biallelic Nf2 mutation in the mouse promotes manifestations of human neurofibromatosis type 2. Genes Dev 13:1617-1630

44. Petrilli AM, Fernandez VC (2016) Role of Merlin/NF2 inactivation in tumor biology. Oncogene 5:537-548

Publisher's Note Springer Nature remains neutral with regard to jurisdictional claims in published maps and institutional affiliations. 